\title{
Antenna Efficiency Measurement from Quality Factor Estimation in Reverberation Chamber
}

\author{
P. Besnier ${ }^{1}$, J. Sol ${ }^{1}$, A. Presse ${ }^{2}$, C. Lemoine ${ }^{1}$, A.-C. Tarot $^{2}$ \\ UMR CNRS 6164, Institut d'Electronique et de Telecommunications de Rennes \\ ${ }^{1}$ INSA de Rennes, ${ }^{2}$ Universite de Rennes 1, Rennes, France \\ Email: philippe.besnier@insa-rennes.fr
}

\begin{abstract}
This communication deals with a method that enables to extract antenna efficiency estimation from reverberation chamber measurements and more specifically from the composite Q-factor estimation of the reverberation chamber. It is another variant of antenna efficiency measurement method in a reverberation chamber that takes advantage of the properties of the composite Q-factor that involves various components including the quality factor of antennas themselves. We show that in a well-overmoded cavity, the Q-factor measured from two antennas in the chamber gives access to the contrast of their efficiency. Estimation uncertainty is discussed with regard to the substitution method.
\end{abstract}

\section{INTRODUCTION}

Antenna efficiency is a key parameter for assessing the power balance of a communication link and for the calibration of antennas in electromagnetic measurements including EMC. Various proposals have been published in the last decade to retrieve antenna efficiency from measurements in a reverberation chamber (RC). In 2001, Hallbjorner discussed the antenna efficiency measurement technique based on the reflection coefficient of a unique antenna. This technique was proposed as a substitution for the technique using the transmission between two antennas [1]. However a reference antenna has to be measured at first, since the measurement is relative to the RC losses. Other authors have proposed an alternative measurement method that gives an absolute value of the radiation efficiency from the estimation of two different Q-factors, one in the frequency domain (steady state) and the other one in the time domain. Indeed, the time domain estimation of the $\mathrm{Q}$ factor is only related to the decay constant of the reverberation chamber [2], [3]. Another technique using $\mathrm{Q}$ estimation in the time domain and the average received power at an antenna terminals was also proposed, requiring either two identical antennas or a kwown antenna [4]. The above mentioned methods rely on the quality of estimation of one or two random variables, typically the average received power by an antenna and / or the quality factor. In this communication, we still suppose that a reference antenna exists. We introduce a new way of assessing the efficiency of an unkwown antenna, from the comparison of the quality factor measured by each antenna in the RC. We show that the efficiency of an unkwown antenna may be retrieved from the hypothesis that the intrisic quality factor of the RC does not depend on the type of antennas located in the chamber. In other words, the quality factor sensed by any antenna

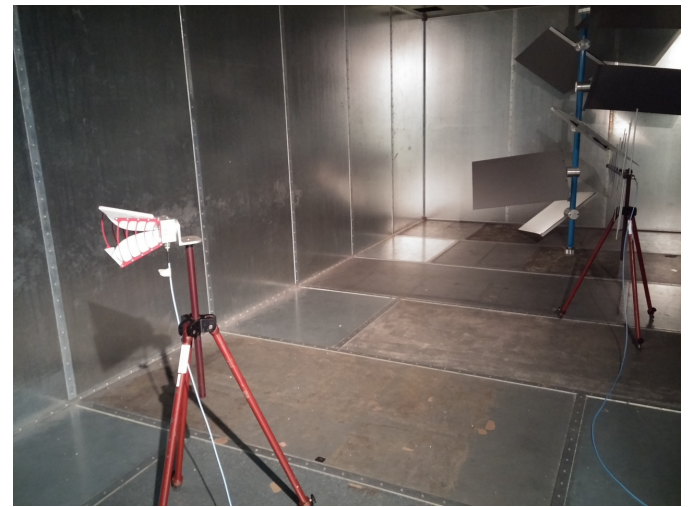

Figure 1. View of the IETR RC. Set-up of section III. B.

in the $\mathrm{RC}$ is identical, as long as the number of antennas is kept constant and without modifcations of their terminal loads. It enables a simple one phase measurement using the only acquisition of backscattering parameters. Section II is dedicated to a brief explanation of the Q-factor measurement procedure with only one antenna [5] highlighting the similarity of Q-factors simultenaously extracted from two antennas in a RC. Section III provides a description of the method and experimental results are provided in section IV.

\section{COMPosite QUALITY FACTOR MEASUREMENTS}

\section{A. Two $Q$-factor estimations with two antennas in a $R C$}

We suppose that two antennas are installed in the chamber (see Fig. 1), and connected to two ports of a vector network analyzer (VNA). We use each of these two antennas separately to retrieve the composite Q-factor from either $S_{11}$ or $S_{22}$ measurements. We will refer to any of these two antennas with the letter $\mathrm{x}$, i.e. $\mathrm{x}=1$ or $\mathrm{x}=2$. The transmitted power from an antenna writes as:

$$
P_{t}=\left(1-\left|\left\langle S_{x x}\right\rangle\right|^{2}\right) \eta_{x} P_{i n j} .
$$

In this equation one identifies $P_{i n j}$ as the arbitrary level of the injected power from the internal source of a VNA. $S_{x x}$ is the scattered energy that goes back (due to mismatch) to the input port of antenna $x$ whose radiation efficiency is $\eta_{x}$. The notation \langle\rangle stands for an ensemble average over the states of the chamber during the stirring process. 
However, the $S_{x x}$ complex parameter may be put in the form of an addition of two contributions. The first one is the free space reflection coefficient and the second one is the backscattering response of the chamber. The first parameter is estimated from the ensemble average of all complex-valued $S_{x x}$ measurements as stated in Eq. 1. The second one is proportional to the complex transfer function of the chamber, denoted by $H$. Thus, a given $S_{x x}$ parameter for any chamber state may be written as:

$$
S_{x x}=\left\langle S_{x x}\right\rangle+\left(1-\left|\left\langle S_{x x}\right\rangle\right|^{2}\right) H \eta_{x} .
$$

Rearranging and taking the square modulus of this equation, we have:

$$
\left|S_{x x}-\left\langle S_{x x}\right\rangle\right|^{2}=\left(1-\left|\left\langle S_{x x}\right\rangle\right|^{2}\right)^{2}|H|^{2} \eta_{x}^{2} .
$$

Then, evaluating the ensemble average of this equality, we obtain:

$$
\left\langle\left|S_{x x}-\left\langle S_{x x}\right\rangle\right|^{2}\right\rangle=\left(1-\left|\left\langle S_{x x}\right\rangle\right|^{2}\right)^{2}\left\langle|H|^{2}\right\rangle \eta_{x}^{2} .
$$

$|H|^{2}$ is homogeneous to the ratio between the received power at an ideally efficient and perfectly matched antenna, denoted by $P_{\text {recI }}$ and the transmitted power in the chamber:

$$
|H|^{2}=\frac{P_{r e c I}}{P_{t}} .
$$

Over all chamber states we may write:

$$
\left\langle|H|^{2}\right\rangle=\frac{\left\langle P_{\text {recI }}\right\rangle}{P_{t}}
$$

The received power at an ideally efficient and perfectly matched transmitting (simultaneously receiving) antenna $P_{r e c I}$ appears to be :

$$
\left\langle P_{\text {recI }}\right\rangle=\frac{\lambda^{2}}{4 \pi} \frac{E^{2}}{Z_{0}} .
$$

The equivalent surface of the transmitting antenna in the chamber is indeed $\lambda^{2} / 4 \pi$. We may now establish the composite Q-factor estimation from the following expression:

$$
Q_{1 a n t}=\left\langle\left|S_{x x}-\left\langle S_{x x}\right\rangle\right|^{2}\right\rangle \frac{Z_{0} \omega \epsilon V}{\left(\lambda^{2} / 4 \pi\right)\left(1-\left|\left\langle S_{x x}\right\rangle\right|^{2}\right)^{2} \eta_{x}^{2}} .
$$

\section{B. Q-factor estimations with two similar antennas}

In a first set of experiments we locate two similar antennas in the chamber, namely two log-periodic antennas in the [2002000] $\mathrm{MHz}$ frequency range.The two log-periodic antennas are installed in the chamber in such a way that their direct coupling is much lower than their average coupling due to the diffuse energy. Using a VNA, the two ports of which being connected to the antenna input ports, the frequency response is recorded into a sequence of 20001 points with a $40 \mathrm{kHz}$ frequency step. The ensemble average is performed over a 10 $\mathrm{MHz}$ frequency band consisting of 250 equally spaced points. The ensemble average is therefore performed over 30 stirrer positions times 250 frequency measurements of the considered complex-valued $S_{11}$ and $S_{22}$ parameters. The two estimations available from each antenna separately, are carried out using

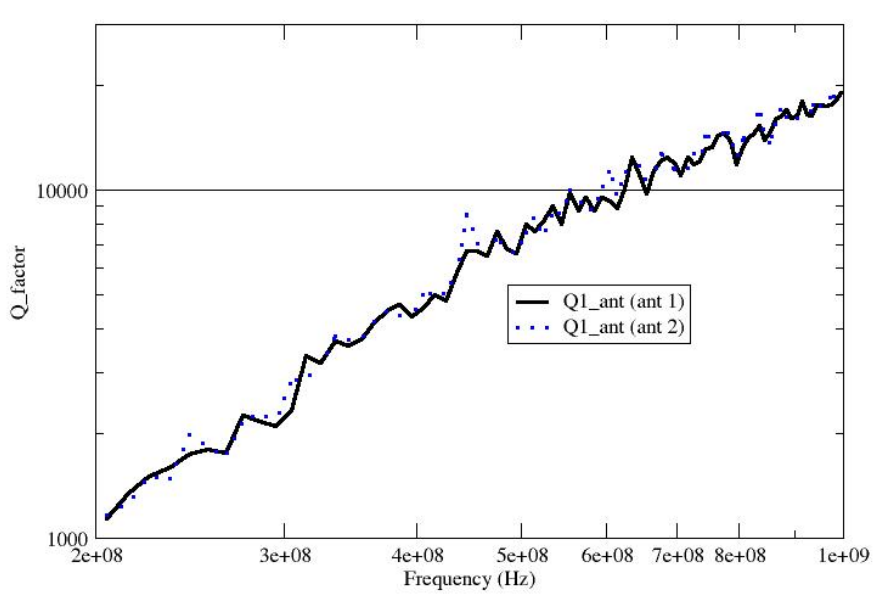

Figure 2. Two Estimations of Q-factor using two similar log-periodic antennas in a RC.

Eq. 8. Note that ensemble average is performed in two steps. A Q-factor estimation is performed for each frequency over all stirrer positions. Then, the final estimation is the average of Q-factors over frequencies. Results are reported in Fig. 2. Both Q-estimation are quite close from each other. From the set of 80 points per curve in the [200-1000] $\mathrm{MHz}$ frequency range, the average relative difference between the two measurements is $1.6 \%$ with a standard deviation of $6.7 \%$. Since the (intrinsic) Q-factor of the chamber is inversely proportional to the square efficiency of the antenna used to measure it, this relative difference provides a good indication of the sensitivity to a change of antenna efficiency as evidenced in the next section.

\section{FROM Q ESTIMATIONS TO EFFICIENCY ESTIMATION}

We now suppose that antenna 1 has a known efficiency $\eta_{1}$ whereas the unknown efficiency $\eta_{2}$ of antenna 2 has to be determined. From Eq. 8, we may now write:

$$
Q_{x}=\frac{Q_{x}^{\#}}{\eta_{x}^{2}}
$$

$Q_{x}^{\#}$ may be defined as the non intrinsic quality factor of the RC that includes the antenna $x$ losses (i.e. conductive or dielectric losses). Since the effective area of an antenna does not depend on the type of (matched) antenna, we have:

$$
\eta_{2}=\sqrt{\frac{Q_{2}^{\#}}{Q_{1}^{\#}}} \eta_{1}=\sqrt{\frac{Q_{2}^{\#}}{Q_{1}}}
$$

Therefore, backscattering information recorded simultenaously at two antenna ports provide a direct estimation of the efficiency of one of these antennas. 


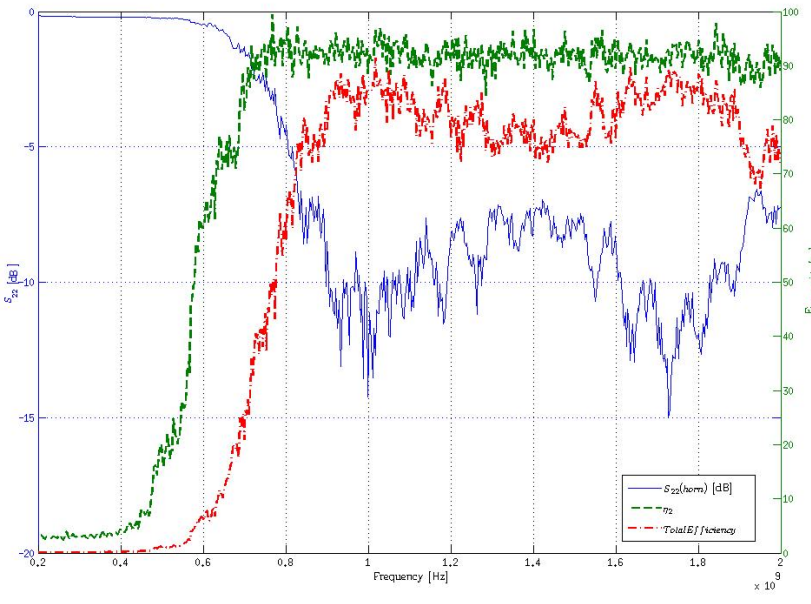

Figure 3. Reflection coefficient, radiation and total efficiency of an horn antenna obtained with the Q-method.

\section{VALIDATION}

All experiments have been carried out in the IETR large reverberation chamber whose dimensions are $2.9 \mathrm{~m} \times 3.7 \mathrm{~m}$ x $8.7 \mathrm{~m}$ (Fig. 1). The frequency of its first natural mode is 43 $\mathrm{MHz}$.

\section{A. Comparison with a standard substitution method}

The proposed approach is compared with a standard method [6] which consists in substituting the antenna under test for a reference antenna whose radiation efficiency is supposed to be known. This is therefore a two-step procedure. The efficiency of the antenna under test (AUT) is characterized once the reference antenna is measured. The estimation of the efficiency is performed with the following computation:

$$
\eta_{2}=\eta_{1} \frac{\left\langle\left|S_{21_{\text {aut }}}\right|^{2}\right\rangle\left(1-\left|\left\langle S_{11_{\text {ref }}}\right\rangle\right|^{2}\right)\left(1-\left|\left\langle S_{22_{\text {ref }}}\right\rangle\right|^{2}\right)}{\left\langle\left|S_{21_{\text {ref }}}\right|^{2}\right\rangle\left(1-\left|\left\langle S_{11_{\text {aut }}}\right\rangle\right|^{2}\right)\left(1-\left|\left\langle S_{22_{\text {aut }}}\right\rangle\right|^{2}\right)} .
$$

In this equation " 1 " and " 2 " refer to port 1 and 2 of a VNA. One of these ports, say port 1, is connected to the reference antenna. The second one is connected first to a similar reference antenna (subscript ref) and then to the AUT (subscript aut) with unknown radiation efficiency $\eta_{2}$.

In what follows, one of the log-periodic antennas introduced in Section II-B is used a reference and both of them are used for the substitution method.

\section{B. Efficiency measurement of a horn antenna}

A horn antenna (ETS-Lindgren 3115A) is used as an AUT within the [200-2000] MHz frequency band of the log-periodic antenna(s). The frequency bandwidth used for frequency stirring is $3 \mathrm{MHz}$ (15 points with a frequency step of $200 \mathrm{kHz}$ ) and 100 stirrer positions are covered. The result obtained from our proposed Q-method is presented in Fig. 3.

As expected the reflection coefficient exhibits a high-pass filter behaviour since the horn antenna is matched only beyond

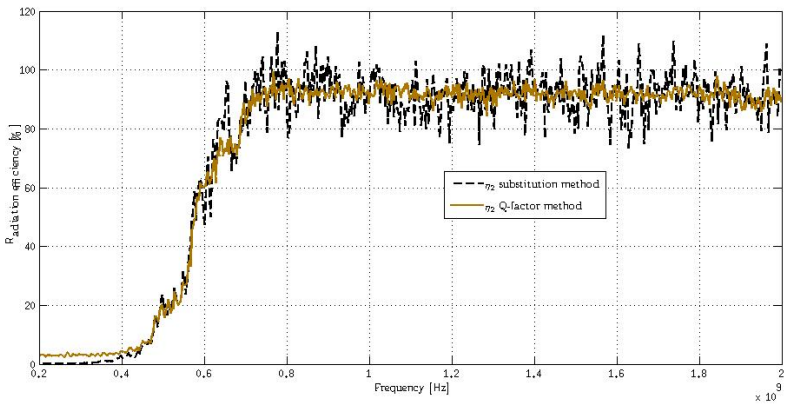

Figure 4. Radiation efficiency of an horn antenna obtained with the Q-method and the substitution method.

$800 \mathrm{MHz}$. In the range $800 \mathrm{MHz}$ to $2 \mathrm{GHz}$ (the lower range of this antenna bandwidth), fluctuations of the reflection coefficient are correlated to those of the total efficiency. On the contrary, the radiation efficiency appears to be approximately independent of the frequency of operation beyond $800 \mathrm{MHz}$. The radiation efficiency of the same antenna with identical stirring procedure is then measured with the substitution method. Both results are compared in Fig. 4. A similar trend is observed which confirms that the two methods correctly retrieve the radiation efficiency of the EUT. Uncertainties associated with the substitution method are however much higher than those of the Q-method, inducing artefacts for such high-efficiency antennas. The Q-method takes profit of two main features. On the one hand all parameters are measured simultaneously. On the other hand, the efficiency is retrieved from the simple ratio of the two non-intrisic quality factors, estimated with a very reasonable uncertainty (see section II-B). Therefore this method appears to be well fitted for analysis of wideband antennas. In the following, we examine its performance as regard a narrowband antenna.

\section{Efficiency measurement of an electro-textile narrowband antenna}

The measured antenna is a planar F-inverted antenna (PIFA) that uses a flexible silicon rubber substrate. Conductive parts of the antenna are composed of electro-textiles. This antenna (5) is designed to operate at a central resonant frequency of $401 \mathrm{MHz}$ for ARGOS applications. Nonetheless, this antenna is measured in the [200-600] MHz frequency band. The frequency bandwidth used for frequency stirring is still 3 $\mathrm{MHz}$ (15 points with a frequency step of $200 \mathrm{kHz}$ ) and 100 stirrer positions are covered. Results are reported in Fig. 6. The analysis of the reflection coefficient confirms that the antenna resonance lies between 400 and $410 \mathrm{MHz}$. Frequency stirring (over $3 \mathrm{MHz}$ ) is obviously associated with some loss of resolution, since it acts as a low-pass filter. Radiation efficiency tends to keep a reasonable level well above its resonance frequency but the total efficiency is rather small out of the vicinity of the resonance, due to mismatch.

This result is then compared to the substitution method. Moreover, the efficiency of the antenna is simulated with a 


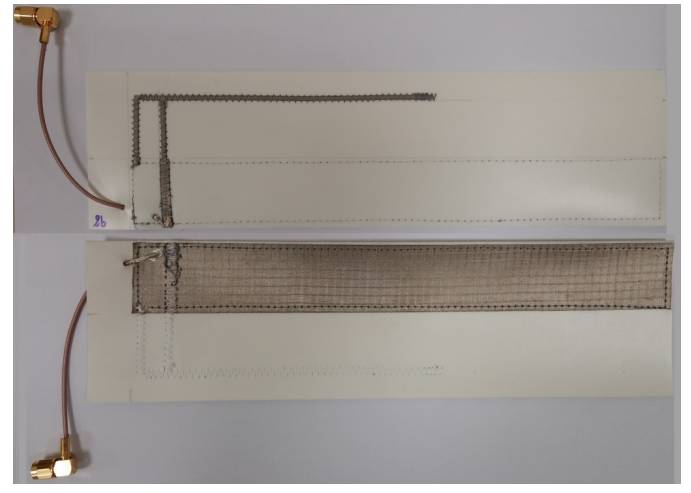

Figure 5. Photography of the ARGOS PIFA textile antenna.

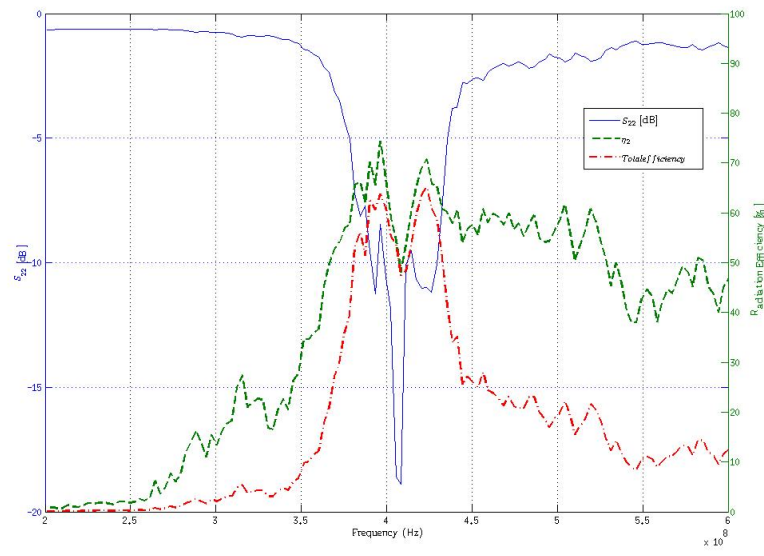

Figure 6. Reflection coefficient, radiation and total efficiency of a textile antenna obtained with the Q-method

Maxwell equation full-wave solver. To do so, we elected a time domain solver based on the finite integration technique (CST). This allows to generate the response over a large frequency bandwidth with a unique simulation. Fig. 7 includes 3 curves. The brown curve represents the radiation efficiency evaluated from the current method. The black curve shows the result obtained from the substitution method. Though their general trend is similar, it highlights once again that uncertainties remains at a lower level if the radiation efficiency is evaluated from the Q-method. Comparison with the simulation result confirms that the radiation efficiency remains at a quite high level well above the PIFA resonance. The simulation curve depicts a slow decrease of efficiency with some smooth fluctuations with frequency in the [400-600] $\mathrm{MHz}$ frequency range. None of the measurements curve exhibits these smooth fluctuations but a monotonic behaviour in terms of a slow decrease of radiation efficiency with frequency. The statistical fluctuations associated with measurements may hide this behaviour especially at frequencies for which antennas are poorly matched whereas radiation efficiency remains significant. The fluctuations of $S_{22}$ estimation in this [400-600] MHz frequency region seems to confirm this hypothesis.

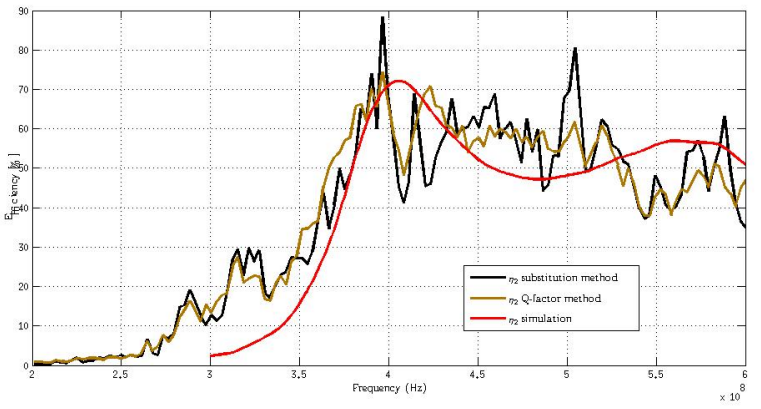

Figure 7. Reflection coefficient, radiation and total efficiency of a textile antenna obtained with the Q-method

\section{CONClusion}

This communication was dedicated to the introdution of a measurement technique to retrieve the radiation efficiency of an antenna in a reverberation chamber. The method is based on a universal property of reverberation chamber as far as it is operated in a well-overmoded regime. In this case, two matched antennas in the chamber receive the same average power whatever their type and position in the chamber. Should they have different radiation efficiencies, this would be not the case anymore. Though the intrinsic Q-factor of the RC remains identical, the ratio of the received power is dependent of their contrast in terms of radiation efficiency. In practice, antennas are not perfectly matched and this is compensated for. The independent estimation of the Q-factor seen by each antenna, using the only set of complex reflection coefficients, leads to a simple efficiency estimation. This method has been compared to a substitution method and exhibits a much better uncertainty budget. Its validity has been succesfully checked with a wideband antenna and a narrowband antenna. Less favorable uncertainty budgets may however appear for out-of-band frequency analysis. Future investigations will be dedicated to further reduction of uncertainty budgets.

\section{REFERENCES}

[1] P. Hallbjorner, Reflective antenna efficiency measurements in reverberation chambers, Microwave Opt. Tecnol. Lett, vol. 30, no. 5, pp 332-335, Sept 2001.

[2] C. L. Holloway, H. A. Shah, R. J. Pirkl, W. F. Young, D. A. Hill and J. Ladbury, Reverberation Chamber Techniques for Determining the Radiation and Total Efficiency of Antennas, IEEE Transactions on Antennas and Propagation, vol. 60, pp. 1758-1770, Apr. 2012.

[3] Chong Li, Tian-Hong, Loh Zhi, Hao Tian, Qian Xu and Yi Huang, A Comparison of Antenna Efficiency Measurements Performed in Two Reverberation Chambers Using Non-reference Antenna Methods, Loughborough Antennas and Propagation Conference (LAPC), 2015.

[4] H.-G. Krauthauser and M. Herbrig, Yet Another Antenna Efficiency Measurement Method in Reverberation Chambers, Electromagnetic Compatibility (EMC), IEEE International Symposium on, pp 536-540, 2010.

[5] P. Besnier, C. Lemoine and J. Sol, Various Estimations of Composite Qfactor with Antennas in a Reverberation Chamber, Electromagnetic Compatibility (EMC), IEEE International Symposium on / EMC EUROPE , pp 1223-1227, 2015.

[6] K. Rosengren and P.-S. Kildal, Radiation efficiency, correlation, diversity gain and capacity of a six-monople antenna array for a MIMO system: theory, simulation and measurement in reverberation chamber, IEE Proc. Microwave Antennas propagation, vol. 152, pp 7-16, 2005. 